Article

\title{
On the Determination of Meshed Distribution Networks Operational Points after Reinforcement
}

\author{
Vasiliki Vita $^{1, *}$, Stavros Lazarou ${ }^{1}$ (), Christos A. Christodoulou ${ }^{1}$ and George Seritan ${ }^{2}$ \\ 1 Department of Electrical and Electronic Engineering Educators, A.S.PE.T.E.-School of Pedagogical and \\ Technological Education, N. Xeraklion, 14121 Athens, Greece \\ 2 Faculty of Electrical Engineering, University Politehnica of Bucharest, Spaiul independentei 313, RO-060042 \\ Bucharest, Romania \\ * Correspondence: vasvita@aspete.gr; Tel.: +30-210-289-6955
}

Received: 25 July 2019; Accepted: 22 August 2019; Published: 25 August 2019

\begin{abstract}
This paper proposes a calculation algorithm that creates operational points and evaluates the performance of distribution lines after reinforcement. The operational points of the line are probabilistically determined using Monte Carlo simulation for several objective functions for a given line. It is assumed that minimum voltage at all nodes has to be balanced to the maximum load served under variable distributed generation production, and to the energy produced from the intermittent renewables. The calculated maximum load, which is higher than the current load, is expected to cover the expected needs for electric vehicles charging. Following the proposed operational patterns, it is possible to have always maximum line capacity. This method is able to offer several benefits. It facilitates of network planning and the estimation of network robustness. It can be used as a tool for network planners, operators and large users. It applies to any type of network including radial and meshed.
\end{abstract}

Keywords: distribution networks; meshed systems; reinforcement; power flow

\section{Introduction}

Medium and low voltage distribution networks are mostly operated as radial; however, in some exceptional cases, they can be connected as meshed or in loops [1]. Radial circuits have many advantages over networked circuits, including easier fault current protection, lower fault currents over most of the circuit, easier voltage control, easier prediction and control of power flows and lower installation cost [2]. On the other hand, meshed networks demonstrate performance improvements and efficiency increase. The reinforcement of already existing distribution networks is an issue of priority for network operators, in an effort to ensure the uninterruptable power supply. Several works have been developed to investigate reinforcement issues in radial and meshed distribution level and to provide adequate simulation tools and methods. Alvarez-Herault et al. [3] demonstrated the benefits of meshing the network instead of reinforcing it. Novoselnik et al. [4] provided a procedure to improve networks' performance taking into consideration the advantage of its meshed development. The networks operate in radial mode even if they are built as meshed. This article proposes a control method to optimally rearrange the radial network. Nevertheless, in this case the network continues to operate radially. Moreover, several planning methods take into consideration that optimal solutions can lead to meshed networks [5], even if they are weakly meshed. Recently, optimization for distribution network planning has led to substantial research activity even if part of the researcher community still supports, to a certain degree, the benefits of distribution system radial operation [6].

Added to the above, the increasing use of electric vehicles and the consequent impact on the operation of the network has to be taken into consideration. Note that the penetration level of electric 
vehicles is higher in the countries where the appropriate infrastructure is widely available [7]. Hence, their charging and their interaction in general with the electricity network can be controlled remotely in a safe manner, maybe by a charging service provider (CSP) [8,9] or on individual vehicle basis [10]. Given the already major resources provided to build the infrastructure, their charging can be prioritized on grid requirements. This would on one hand meet possible infrastructure constraints and on the other hand improve electricity system performance, even though new significant load is added. Furthermore, this procedure would offer time for system reinforcements, if these are needed.

However, system inertia exists in the system, and hence, controlled charging has to be achieved gradually [11]. It can start from lower penetration of electric vehicles and random charging when needed, up to the level where electric vehicles would charge only when grid has the essential capacity to allocate in this exercise. In this way, electric vehicles are behaving as battery storage [12]. The proposed procedure would also be enhanced in the future from improved battery performance connected to enhanced electric vehicle range that would reduce driver anxiety. However, in any case, this approach could decrease vehicle owners' satisfaction who are used to a continued service currently offered from fossil fuel powered vehicles. This approach could also possibly deteriorate battery condition. Of course, on the other hand, drivers' behavior affects, in a different manner, electricity systems [13].

Additionally, it could create discomfort to the industry [14] that is going to implement the incentive, but the benefits are more important to the effort. It has to be mentioned that electricity companies already have the experience in operating demand side management programs, restricted by the limited communication resources of the past. These demand side management programs are not considered as suitable for electric vehicles optimal charging [15]. Building upon this direction, bibliography proposes electric vehicle charging regulation that is organized on the transmission system or to the level of the distribution network based on a range of decision factors [16] that have to do with operational conditions or production from intermitted renewables.

Having mentioned the above, electric vehicles are to be charged in a way to provide always the necessary load [17]. This approach would contribute in achieving grid operational optimization as it is also projected in this study. This optimization would be based on the incoming intermittent energy from renewables connected to the distribution grid and the voltage profile of the line under investigation.

Except from the electric vehicles, the already widely developed connection of distributed generation [18] affects the operation of the grid. It creates bidirectional power flows [19] and changes network topology. As expected, network planning with distributed generation research applies similar to meshed network planning optimization methods [20,21]. Meshed planning in conjunction to the connection of distributed generation could potentially enhance network's operation [22]. It has to be mentioned that in our work all components are assumed as functioning; however, in practice there could be malicious and faulty units [23].

Several optimization methods are being used for distribution network analysis, including Tabu search (TS), Simulated annealing (SA), Genetic algorithm (GA), Evolutionary strategy (ES), Artificial immune system (AIS), Ant colony optimization (ACO), Ant colony system (ACS), Particle swarm optimization (PSO) and Hybrid TS/GA (Memetic) [24]. This research is based on the Monte Carlo method [25], which is implemented to a plethora of applications [26], including network planning. On power systems, it is widely used to simulate probabilistic phenomena [27] in general, and power flows for steady state simulations [28]. Active distribution [29] and expansion [30] planning under uncertainty remains an issue of paramount importance. The dynamic programming method is a viable alternative [31]. Monte Carlo is frequently the method of choice to this direction [32]. It requires, even for today's standards, high computational power; hence, for the simulations of this work, Okeanos cloud computing [33] is used, which is a supercomputing facility, organized on virtual machines available to the research community.

All the proposed methods for distribution network planning seek to optimize a value directly related to grids' operation. This could be total installation and operational cost, minimum power or copper losses or voltage deviation. The proposed solutions up to today offer a deterministic approach 
towards solving the given electric vehicle connection capacity problem. However, they lack the capability to enhance user's decision making capability offering additional information of system robustness, behavior and performance after reinforcement. This could be a useful tool for engineers who engage in network planning, operation and large users of the system. In this paper, a calculation procedure is developed in an effort to upgrade the operation of distribution grids, providing benefits in terms of their optimal point of operation that can be used for demand management purposes. It can be complimentary to any other optimization method for distribution grid planning. Moreover, it aims to demonstrate that distribution medium voltage network is better if operated as meshed and to provide solutions in terms of network planning. Its main contribution is the application of a novel method, based on Monte Carlo and objective functions to evaluate decisions for network reinforcement. This research is organized in three sections. Section 2. 'System under examination and problem formulation' describes the network under investigation and the procedure that it was followed to tackle the research question. Section 3, 'Results and Discussion' provides the outcome of our analysis and its commenting. Section 4 contains the conclusions and future work.

\section{System under Examination and Problem Formulation}

Tables 1-3 present the technical characteristics and the configuration of the distribution network under study [34]. An abstract graphical representation of the network is provided at Figure 1. The voltage level of the grid is $20 \mathrm{kV}$, the total length is $55 \mathrm{~km}$ and the installed capacity is 12 MVA. The network includes $4520 / 0.4 \mathrm{kV}$ distribution transformers and 24 renewable energy sources plants (photovoltaics). The resistance and the reactance of the conductors is $R=1.268 \Omega / \mathrm{km}$ and $X=0.422 \Omega / \mathrm{km}$ for ACSR $16 \mathrm{~mm}^{2}, \mathrm{R}=1.071 \Omega / \mathrm{km}$ and $X=0.393 \Omega / \mathrm{km}$ for ACSR $35 \mathrm{~mm}^{2}$ and $\mathrm{R}=0.215 \Omega / \mathrm{km}$ and $X=0.334 \Omega / \mathrm{km}$ for ACSR $95 \mathrm{~mm}^{2}$. In order to improve the performance of this line [34] and to demonstrate the capability of the proposed method suitable for meshed networks, it is being reinforced in a meshed manner, connecting the nodes 51 and 84 using ACSR $95 \mathrm{~mm}^{2}$ conductors. The distance between these nodes is $8 \mathrm{~km}$, creating a resistance of $1.72 \Omega$ and impedance of $2.672 \Omega$, or pu resistance and impedance 0.43 pu and $0.668 \mathrm{pu}$, respectively.

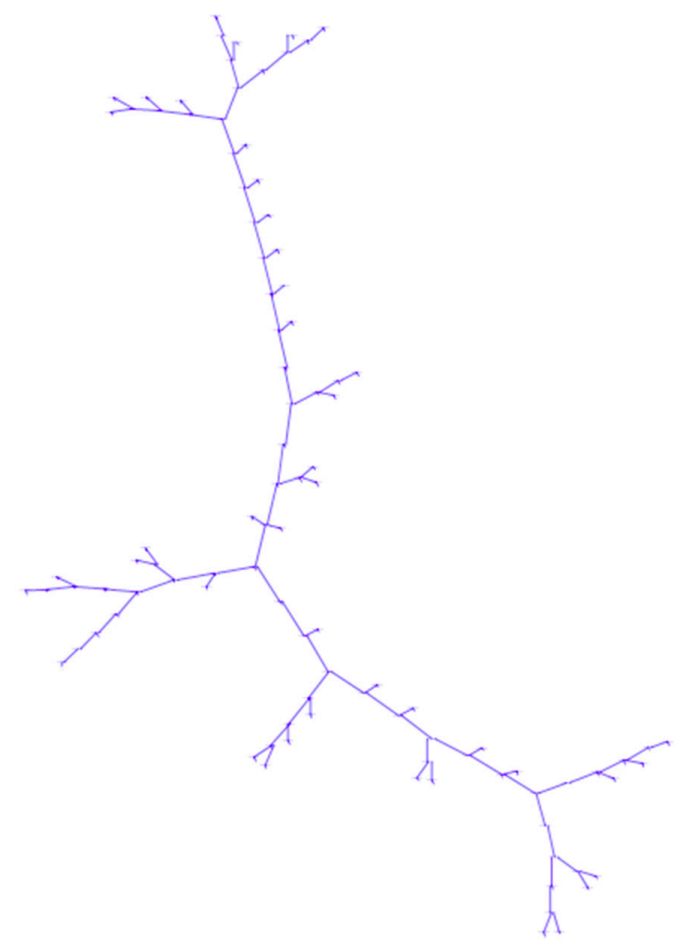

Figure 1. Abstract graphical representation of the network. 
Table 1. Network topology.

\begin{tabular}{|c|c|c|c|c|c|}
\hline $\begin{array}{c}\text { Line } \\
\text { (Node } \rightarrow \text { Node) }\end{array}$ & Length [km] & $\begin{array}{c}\text { Cross Section } \\
{\left[\mathrm{mm}^{2}\right]}\end{array}$ & $\begin{array}{c}\text { Line } \\
\text { (Node } \rightarrow \text { Node) }\end{array}$ & Length [km] & $\begin{array}{c}\text { Cross Section } \\
{\left[\mathrm{mm}^{2}\right]}\end{array}$ \\
\hline $1 \rightarrow 2$ & 0.25 & 95 & $57 \rightarrow 58$ & 0.483 & 35 \\
\hline $1 \rightarrow 3$ & 2.305 & 95 & $58 \rightarrow 59$ & 0.677 & 35 \\
\hline $2 \rightarrow 4$ & 0.488 & 95 & $58 \rightarrow 60$ & 0.014 & 16 \\
\hline $4 \rightarrow 5$ & 0.945 & 35 & $61 \rightarrow 62$ & 0.5 & 95 \\
\hline $6 \rightarrow 4$ & 0.505 & 95 & $61 \rightarrow 63$ & 0.1 & 95 \\
\hline $6 \rightarrow 7$ & 0.333 & 95 & $64 \rightarrow 63$ & 0.648 & 16 \\
\hline $6 \rightarrow 8$ & 0.132 & 16 & $65 \rightarrow 63$ & 0.1 & 95 \\
\hline $7 \rightarrow 9$ & 0.473 & 16 & $65 \rightarrow 66$ & 0.18 & 16 \\
\hline $7 \rightarrow 10$ & 1 & 95 & $66 \rightarrow 67$ & 0.524 & 16 \\
\hline $10 \rightarrow 11$ & 0.55 & 95 & $66 \rightarrow 68$ & 0.169 & 16 \\
\hline $11 \rightarrow 12$ & 0.179 & 95 & $68 \rightarrow 117$ & 0.22 & 16 \\
\hline $11 \rightarrow 13$ & 0.068 & 35 & $68 \rightarrow 69$ & 0.014 & 16 \\
\hline $11 \rightarrow 14$ & 0.242 & 95 & $70 \rightarrow 71$ & 0.507 & 16 \\
\hline $14 \rightarrow 15$ & 0.521 & 95 & $70 \rightarrow 72$ & 0.008 & 35 \\
\hline $14 \rightarrow 16$ & 0.363 & 16 & $73 \rightarrow 74$ & 0.01 & 35 \\
\hline $15 \rightarrow 17$ & 0.6 & 95 & $75 \rightarrow 76$ & 0.01 & 35 \\
\hline $17 \rightarrow 18$ & 0.225 & 16 & $75 \rightarrow 77$ & 0.01 & 35 \\
\hline $17 \rightarrow 19$ & 0.197 & 95 & $78 \rightarrow 79$ & 0.451 & 35 \\
\hline $19 \rightarrow 20$ & 0.208 & 95 & $78 \rightarrow 80$ & 0.043 & 35 \\
\hline $20 \rightarrow 21$ & 0.008 & 35 & $80 \rightarrow 81$ & 0.479 & 35 \\
\hline $20 \rightarrow 22$ & 0.006 & 95 & $82 \rightarrow 83$ & 0.571 & 35 \\
\hline $22 \rightarrow 23$ & 0.334 & 95 & $82 \rightarrow 81$ & 0.01 & 35 \\
\hline $22 \rightarrow 24$ & 0.785 & 35 & $84 \rightarrow 85$ & 1.384 & 35 \\
\hline $25 \rightarrow 26$ & 2.419 & 35 & $84 \rightarrow 86$ & 0.022 & 35 \\
\hline $26 \rightarrow 27$ & 3.125 & 35 & $87 \rightarrow 88$ & 0.075 & 35 \\
\hline $26 \rightarrow 28$ & 0.258 & 35 & $89 \rightarrow 84$ & 0.244 & 35 \\
\hline $29 \rightarrow 30$ & 0.871 & 16 & $90 \rightarrow 82$ & 0.266 & 16 \\
\hline $30 \rightarrow 31$ & 0.14 & 16 & $90 \rightarrow 91$ & 0.4 & 16 \\
\hline $32 \rightarrow 33$ & 0.304 & 95 & $90 \rightarrow 92$ & 0.06 & 16 \\
\hline $32 \rightarrow 34$ & 0.311 & 16 & $93 \rightarrow 94$ & 4.968 & 16 \\
\hline $32 \rightarrow 35$ & 0.108 & 95 & $95 \rightarrow 93$ & 0.6 & 16 \\
\hline $35 \rightarrow 36$ & 0.469 & 35 & $96 \rightarrow 95$ & 0.228 & 16 \\
\hline $36 \rightarrow 37$ & 0.465 & 35 & $92 \rightarrow 97$ & 0.359 & 16 \\
\hline $36 \rightarrow 38$ & 0.661 & 95 & $97 \rightarrow 98$ & 0.007 & 16 \\
\hline $38 \rightarrow 39$ & 0.5 & 35 & $99 \rightarrow 97$ & 0.368 & 16 \\
\hline $38 \rightarrow 40$ & 0.447 & 95 & $100 \rightarrow 101$ & 0.018 & 16 \\
\hline $41 \rightarrow 40$ & 0.326 & 16 & $102 \rightarrow 3$ & 0.001 & 95 \\
\hline $42 \rightarrow 43$ & 0.082 & 95 & $103 \rightarrow 104$ & 0.07 & 35 \\
\hline $42 \rightarrow 44$ & 0.3 & 16 & $105 \rightarrow 104$ & 0.198 & 35 \\
\hline $42 \rightarrow 40$ & 0.343 & 95 & $106 \rightarrow 49$ & 0.001 & 35 \\
\hline $45 \rightarrow 46$ & 1.365 & 16 & $107 \rightarrow 83$ & 0.006 & 35 \\
\hline $45 \rightarrow 47$ & 0.023 & 35 & $108 \rightarrow 98$ & 0.01 & 35 \\
\hline $48 \rightarrow 45$ & 1.275 & 16 & $109 \rightarrow 78$ & 0.487 & 35 \\
\hline $46 \rightarrow 49$ & 0.102 & 35 & $110 \rightarrow 28$ & 0.14 & 35 \\
\hline $46 \rightarrow 50$ & 10.181 & 16 & $111 \rightarrow 37$ & 0.298 & 35 \\
\hline $51 \rightarrow 52$ & 0.777 & 35 & $112 \rightarrow 37$ & 0.001 & 35 \\
\hline $52 \rightarrow 53$ & 0.286 & 35 & $113 \rightarrow 80$ & 0.125 & 35 \\
\hline $52 \rightarrow 54$ & 0.702 & 35 & $114 \rightarrow 35$ & 0.277 & 35 \\
\hline $54 \rightarrow 55$ & 0.076 & 35 & $115 \rightarrow 81$ & 0.356 & 35 \\
\hline $54 \rightarrow 56$ & 0.414 & 35 & $116 \rightarrow 87$ & 1.297 & 35 \\
\hline
\end{tabular}


Table 2. Installed capacity $(\mathrm{S})$, maximum and minimum active and reactive loads $\left(\mathrm{P}_{\max }, \mathrm{Q}_{\max }, \mathrm{P}_{\min }\right.$, $\mathrm{Q}_{\mathrm{min}}$ ) of each $20 / 0.4 \mathrm{kV}$ distribution transformer.

\begin{tabular}{cccccccccccc}
\hline Node & $\begin{array}{c}\mathbf{S} \\
{[\mathbf{k V A}]}\end{array}$ & $\begin{array}{c}\mathbf{P}_{\max } \\
{[\mathbf{k W}]}\end{array}$ & $\begin{array}{c}\mathbf{Q}_{\max } \\
{[\mathbf{k V A r}]}\end{array}$ & $\begin{array}{c}\mathbf{P}_{\min } \\
{[\mathbf{k W}]}\end{array}$ & $\begin{array}{c}\mathbf{Q}_{\min } \\
{[\mathbf{k V A r}]}\end{array}$ & $\mathbf{N o d e}$ & $\begin{array}{c}\mathbf{S} \\
{[\mathbf{k V A}]}\end{array}$ & $\begin{array}{c}\mathbf{P}_{\max } \\
{[\mathbf{k W}]}\end{array}$ & $\begin{array}{c}\mathbf{Q}_{\max } \\
{[\mathbf{k V A r}]}\end{array}$ & $\begin{array}{c}\mathbf{P}_{\min } \\
{[\mathbf{k W}]}\end{array}$ & $\begin{array}{c}\mathbf{Q}_{\min } \\
{[\mathbf{k V A r}]}\end{array}$ \\
\hline 1 & 50 & 17 & 10 & 1 & 1 & 46 & 200 & 68 & 42 & 5 & 3 \\
2 & 485 & 164 & 102 & 12 & 7 & 49 & 100 & 34 & 21 & 2 & 1 \\
4 & 720 & 243 & 151 & 17 & 11 & 57 & 210 & 71 & 44 & 5 & 3 \\
6 & 100 & 34 & 21 & 2 & 1 & 59 & 50 & 17 & 10 & 1 & 1 \\
7 & 320 & 108 & 67 & 8 & 5 & 60 & 100 & 34 & 21 & 2 & 1 \\
10 & 920 & 311 & 193 & 22 & 14 & 61 & 75 & 25 & 16 & 2 & 1 \\
14 & 150 & 51 & 31 & 4 & 2 & 64 & 560 & 189 & 117 & 14 & 8 \\
15 & 660 & 223 & 138 & 16 & 10 & 66 & 250 & 85 & 52 & 6 & 4 \\
19 & 75 & 25 & 16 & 2 & 1 & 117 & 410 & 139 & 86 & 10 & 6 \\
20 & 100 & 34 & 21 & 2 & 1 & 69 & 250 & 85 & 52 & 6 & 4 \\
22 & 250 & 85 & 52 & 6 & 4 & 67 & 175 & 59 & 37 & 4 & 3 \\
25 & 1490 & 504 & 312 & 36 & 22 & 12 & 160 & 54 & 34 & 4 & 2 \\
26 & 100 & 34 & 21 & 2 & 1 & 16 & 100 & 34 & 21 & 2 & 1 \\
28 & 50 & 17 & 10 & 1 & 1 & 71 & 160 & 54 & 34 & 4 & 2 \\
27 & 360 & 122 & 75 & 9 & 5 & 73 & 160 & 54 & 34 & 4 & 2 \\
29 & 810 & 274 & 170 & 20 & 12 & 75 & 250 & 85 & 52 & 6 & 4 \\
31 & 100 & 34 & 21 & 2 & 1 & 96 & 160 & 54 & 34 & 4 & 2 \\
9 & 410 & 139 & 86 & 10 & 6 & 94 & 250 & 85 & 52 & 6 & 4 \\
34 & 100 & 34 & 21 & 2 & 1 & 92 & 100 & 34 & 21 & 2 & 1 \\
40 & 100 & 34 & 21 & 2 & 1 & 98 & 160 & 54 & 34 & 4 & 2 \\
41 & 160 & 54 & 34 & 4 & 2 & 99 & 160 & 54 & 34 & 4 & 2 \\
45 & 50 & 17 & 10 & 1 & 1 & 101 & 50 & 17 & 10 & 1 & 1 \\
50 & 545 & 184 & 114 & 13 & 8 & & & & & & \\
\hline
\end{tabular}

Table 3. Renewable energy sources at every node.

\begin{tabular}{cccccc}
\hline Node & $\begin{array}{c}\text { Installed } \\
\text { Power }[\mathbf{k W}]\end{array}$ & Node & $\begin{array}{c}\text { Installed } \\
\text { Power }[\mathbf{k W}]\end{array}$ & Node & $\begin{array}{c}\text { Installed } \\
\text { Power [kW] }\end{array}$ \\
\hline 110 & 100 & 114 & 100 & 109 & 100 \\
72 & 100 & 111 & 100 & 113 & 500 \\
74 & 100 & 112 & 100 & 115 & 20 \\
76 & 100 & 39 & 100 & 107 & 100 \\
21 & 500 & 106 & 100 & 86 & 100 \\
13 & 100 & 47 & 100 & 88 & 1274 \\
103 & 100 & 55 & 470 & 116 & 1815 \\
105 & 100 & 56 & 700 & 108 & 50 \\
\hline
\end{tabular}

The total load of the line is the sum of all $20 / 0.4 \mathrm{kV}$ transformers load and the production of renewables is fed to the line through the renewable energy sources connection points. Renewable energy sources connected to this line are located in the relatively proximity with each other. This is to the comparably limited size of a distribution line such as the one under investigation. Plants' production is considered as analogous to their installed power but the same at each time period across all of the line since solar irradiation can be safely considered as being identical.

Figure 2 depicts the flow chart of the developed calculation procedure. The algorithm creates randomly possible loading for all transformers feeding the low voltage part of the distribution network and perform power flow analysis. Then, the values of the objective functions are calculated, and this algorithm reiterates 4 million times. The above procedure is being repeated for distributed generators production from 0 to full of their installed capacity in 1/10 steps of the maximum value. 


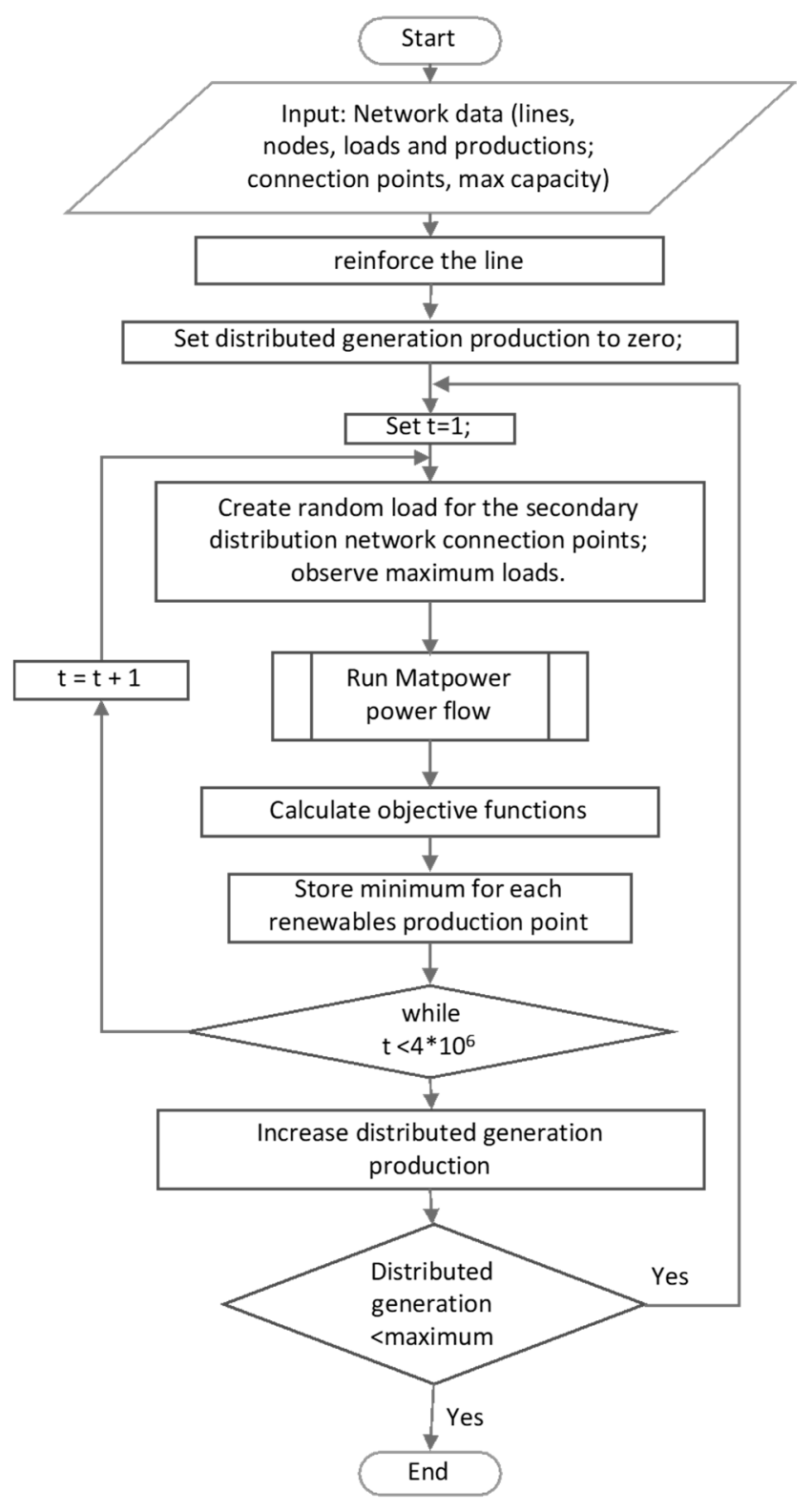

Figure 2. Flow chart of the developed calculation procedure.

AC power flow is used to perform the calculations for this analysis [35], by using an appropriate computer tool $[25,26]$. All nodes are $P Q$, being able to offer active and reactive power, except from the slack node. In this case, there is no generator connected. The distributed generators are simulated as negative loads given that based on research questions, their production is known. The following equations used to simulate the branches [36]:

$$
\left[\frac{\mathrm{i}_{\mathrm{f}}}{\mathrm{i}_{\mathrm{t}}}\right]=\mathrm{Y}_{\mathrm{br}}\left[\frac{\mathrm{v}_{\mathrm{f}}}{\mathrm{v}_{\mathrm{t}}}\right]
$$

where: $v_{f}, v_{t}, i_{f}$ and $i_{t}$ are the terminal voltages and currents.

Equation (1) connects voltages at all nodes and currents. The impedance matrix $\left(\mathrm{Y}_{\mathrm{br}}\right)$ expresses the impedances across all branches forming a table that is unique for each network.

The admittance matrix $Y_{b r}$ is as follows:

$$
Y_{b r}=\left[\begin{array}{ll}
y_{f f}^{i} & y_{f t}^{i} \\
y_{t f}^{i} & y_{t t}^{i}
\end{array}\right]
$$


Eventually, the balance equation based on Kirchhoff's laws can be written as follows [35], f(V, Sg) needs to equal to zero:

$$
\mathrm{f}\left(\mathrm{V}, \mathrm{S}_{\mathrm{g}}\right)=\mathrm{S}_{\text {bus }}(\mathrm{V})+\mathrm{S}_{\mathrm{d}}-\mathrm{C}_{\mathrm{g}} \cdot \mathrm{S}_{\mathrm{g}}
$$

where $S_{g}$, and $S_{d}$ are the generators' and loads' apparent power, then (3) becomes [35] $f_{p}\left(\Theta, V_{m}, P_{g}\right)$ and $f q\left(\Theta, V_{m}, P_{g}\right)$ that also need to equal to zero:

$$
\begin{gathered}
f_{P}\left(\Theta, V_{m}, P_{g}\right)=P_{\text {bus }}\left(\Theta, V_{m}\right)+P_{d}-C_{g} \cdot S_{g} \\
f_{P}\left(\Theta, V_{m}, P_{g}\right)=Q_{\text {bus }}\left(\Theta, V_{m}\right)+Q_{d}-C_{g} \cdot Q_{g}
\end{gathered}
$$

and:

$$
\begin{gathered}
g(x)=\left[\begin{array}{c}
f_{P}^{[i\}}\left(\Theta, V_{m}, P_{g}\right) \\
f_{Q}^{j i\}}\left(\Theta, V_{m}, P_{g}\right)
\end{array}\right] \\
\forall i \in I_{P V} \cup I_{P V}, \forall j \in I_{P Q}
\end{gathered}
$$

where vector $\mathrm{x}$ equals to:

$$
\begin{gathered}
x=\left[\begin{array}{c}
\Theta_{\{l\}} \\
U_{m}^{j j\}}
\end{array}\right] \\
\forall i \notin I_{r e f} \cup I_{P V}, \forall j \in I_{P Q}
\end{gathered}
$$

The objective function to be minimized is:

$$
\mathrm{e}=\mathrm{w}_{1} \cdot \mathrm{P}_{\mathrm{L}}+\mathrm{w}_{2} \cdot \mathrm{V}_{\min }
$$

$\mathrm{P}_{\mathrm{L}}$ stands for the total active load of the line, $\mathrm{V}_{\min }$ is the minimum voltage observed at any node and $\mathrm{w}_{1}$ and $\mathrm{w}_{2}$ are the weight factors. In this work, four different cases are examined according to the following Table 4.

Table 4. Examined cases.

\begin{tabular}{ccc}
\hline Case & $\mathbf{w}_{\mathbf{1}}$ & $\mathbf{w}_{\mathbf{2}}$ \\
\hline 1 & 0.5 & 0.5 \\
2 & 0.6 & 0.4 \\
3 & 0.7 & 0.3 \\
4 & 0.8 & 0.2 \\
\hline
\end{tabular}

\section{Results and Discussion}

Simulation results have shown that in order to achieve the maximum possible total loading without substantially compromising voltages across the line, the transformer that feed the low voltage distribution system has to have the calculated load. Table 5 and Figure 3 show the calculated values for the objective function for each case; the values are increasing when the production from the renewables also increasing, since more load is able to be fed to the low voltage network distribution transformers without decreasing voltage at all points. The production is able to feed nearby loads; hence, the total load served is increasing without severely affecting voltage drop. The higher increase is observed when voltage drop has higher weighting factor for the case 1 and lower for case 4 . The graphical representation of this interrelation shall normally provide perfect curves; however, due to the probabilistic approach applied, there could be minor rounding errors that do not substantially affect the results. The source code for this publication has been written on Matpower 6.0 [36,37] and Mathworks Matlab 2017a [38]. The Monte Carlo simulation was run on Aris high performance computing [39]. 
Table 5. Calculated value of the objective function. DG: distribution generation.

\begin{tabular}{ccccc}
\hline & Case 1 & Case 2 & Case 3 & Case 4 \\
\hline without DG & 0.7683 & 0.7611 & 0.7611 & 0.7689 \\
$\mathbf{1 0 \%}$ of DG prod & 0.7872 & 0.7772 & 0.7759 & 0.7795 \\
$\mathbf{2 0 \%}$ of DG prod & 0.7997 & 0.7918 & 0.7871 & 0.7879 \\
$\mathbf{3 0 \%}$ of DG prod & 0.8097 & 0.8045 & 0.7966 & 0.7949 \\
$\mathbf{4 0 \%}$ of DG prod & 0.8175 & 0.8155 & 0.8048 & 0.8009 \\
$\mathbf{5 0 \%}$ of DG prod & 0.8261 & 0.8251 & 0.8124 & 0.8061 \\
$\mathbf{6 0 \%}$ of DG prod & 0.8351 & 0.8303 & 0.8194 & 0.8107 \\
$\mathbf{7 0 \%}$ of DG prod & 0.843 & 0.8361 & 0.8255 & 0.8148 \\
$\mathbf{8 0 \%}$ of DG prod & 0.8499 & 0.8434 & 0.8309 & 0.8117 \\
$\mathbf{9 0 \%}$ of DG prod & 0.8521 & 0.8483 & 0.8346 & 0.8143 \\
full DG prod & 0.8537 & 0.8496 & 0.8356 & 0.8166 \\
\hline
\end{tabular}

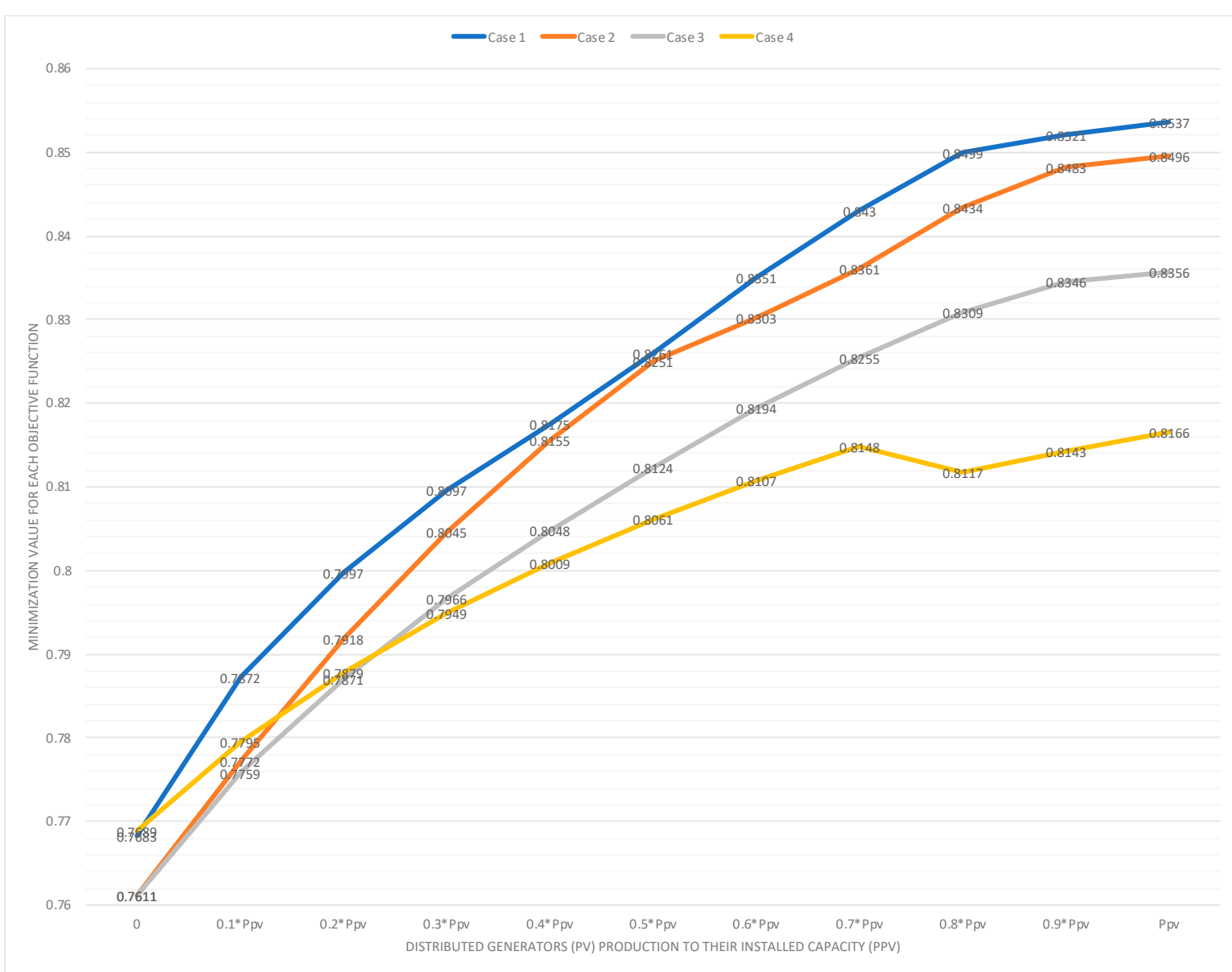

Figure 3. Graphical representation of the calculated values.

Tables 6-9 and their graphical representations Figures 4-6 present the recommended loading per node for each examined case, according to the outcomes of the developed calculation procedures. For the case 1 (Table 6 and Figure 4), the obtained results indicate that most of the transformers can be fed near their installed capacity. However, there are connection points that when energy production from renewables is high, they need to keep their load low and vice versa. Other transformers, such as 49 and 15, need to be downscaled if these operational patterns are to be applied. In all cases, its proposed loading does not exceed $60 \%$ of their installed capacity. Another solution could be to further reinforce the line at these points. 
Table 6. Loading per node for case 1.

\begin{tabular}{|c|c|c|c|c|c|c|}
\hline Node & $0 \%$ DG & $10 \%$ DG & $20 \%$ DG & $30 \%$ DG & $40 \%$ DG & $50-100 \%$ DG \\
\hline 1 & $83.8 \%$ & $3.0 \%$ & $83.8 \%$ & $81.8 \%$ & $39.1 \%$ & $15.2 \%$ \\
\hline 2 & $88.6 \%$ & $90.2 \%$ & $88.6 \%$ & $96.0 \%$ & $94.3 \%$ & $88.5 \%$ \\
\hline 4 & $90.5 \%$ & $91.1 \%$ & $90.5 \%$ & $86.9 \%$ & $98.9 \%$ & $91.9 \%$ \\
\hline 6 & $97.6 \%$ & $92.5 \%$ & $97.6 \%$ & $98.0 \%$ & $92.8 \%$ & $99.2 \%$ \\
\hline 7 & $34.7 \%$ & $94.8 \%$ & $34.7 \%$ & $92.4 \%$ & $92.7 \%$ & $82.6 \%$ \\
\hline 10 & $68.7 \%$ & $97.0 \%$ & $68.7 \%$ & $12.4 \%$ & $83.8 \%$ & $47.8 \%$ \\
\hline 14 & $82.8 \%$ & $45.2 \%$ & $82.8 \%$ & $76.0 \%$ & $18.5 \%$ & $98.8 \%$ \\
\hline 15 & $16.5 \%$ & $61.8 \%$ & $16.5 \%$ & $32.5 \%$ & $27.8 \%$ & $33.0 \%$ \\
\hline 19 & $97.3 \%$ & $84.0 \%$ & $97.3 \%$ & $87.1 \%$ & $84.4 \%$ & $82.8 \%$ \\
\hline 20 & $51.4 \%$ & $79.1 \%$ & $51.4 \%$ & $26.0 \%$ & $84.6 \%$ & $84.2 \%$ \\
\hline 22 & $67.6 \%$ & $91.3 \%$ & $67.6 \%$ & $90.0 \%$ & $86.8 \%$ & $97.1 \%$ \\
\hline 25 & $99.3 \%$ & $91.9 \%$ & $99.3 \%$ & $84.6 \%$ & $71.6 \%$ & $93.2 \%$ \\
\hline 26 & $90.8 \%$ & $91.7 \%$ & $90.8 \%$ & $82.5 \%$ & $94.4 \%$ & $94.0 \%$ \\
\hline 28 & $83.8 \%$ & $78.8 \%$ & $83.8 \%$ & $37.9 \%$ & $28.9 \%$ & $6.7 \%$ \\
\hline 27 & $84.1 \%$ & $54.2 \%$ & $84.1 \%$ & $82.4 \%$ & $91.5 \%$ & $95.7 \%$ \\
\hline 29 & $89.7 \%$ & $94.7 \%$ & $89.7 \%$ & $84.0 \%$ & $31.4 \%$ & $64.4 \%$ \\
\hline 31 & $27.0 \%$ & $61.3 \%$ & $27.0 \%$ & $80.0 \%$ & $82.4 \%$ & $76.7 \%$ \\
\hline 9 & $74.6 \%$ & $39.7 \%$ & $74.6 \%$ & $42.3 \%$ & $28.7 \%$ & $98.0 \%$ \\
\hline 34 & $31.3 \%$ & $90.4 \%$ & $31.3 \%$ & $60.8 \%$ & $92.4 \%$ & $71.1 \%$ \\
\hline 40 & $58.6 \%$ & $57.4 \%$ & $58.6 \%$ & $88.7 \%$ & $75.8 \%$ & $11.2 \%$ \\
\hline 41 & $84.3 \%$ & $88.7 \%$ & $84.3 \%$ & $32.7 \%$ & $68.6 \%$ & $93.9 \%$ \\
\hline 45 & $32.6 \%$ & $82.5 \%$ & $32.6 \%$ & $68.2 \%$ & $54.5 \%$ & $6.7 \%$ \\
\hline 50 & $45.6 \%$ & $43.5 \%$ & $45.6 \%$ & $57.6 \%$ & $54.4 \%$ & $56.9 \%$ \\
\hline 46 & $38.9 \%$ & $3.6 \%$ & $38.9 \%$ & $80.4 \%$ & $19.6 \%$ & $62.0 \%$ \\
\hline 49 & $14.9 \%$ & $16.5 \%$ & $14.9 \%$ & $3.4 \%$ & $30.5 \%$ & $53.3 \%$ \\
\hline 53 & $7.6 \%$ & $64.4 \%$ & $7.6 \%$ & $80.3 \%$ & $92.2 \%$ & $1.5 \%$ \\
\hline 59 & $86.9 \%$ & $44.9 \%$ & $86.9 \%$ & $97.1 \%$ & $49.8 \%$ & $82.2 \%$ \\
\hline 60 & $19.5 \%$ & $30.2 \%$ & $19.5 \%$ & $79.6 \%$ & $22.7 \%$ & $87.9 \%$ \\
\hline 61 & $69.5 \%$ & $95.7 \%$ & $69.5 \%$ & $81.2 \%$ & $61.9 \%$ & $97.8 \%$ \\
\hline 64 & $75.0 \%$ & $12.2 \%$ & $75.0 \%$ & $51.6 \%$ & $28.9 \%$ & $71.5 \%$ \\
\hline 66 & $70.9 \%$ & $62.3 \%$ & $70.9 \%$ & $55.8 \%$ & $70.6 \%$ & $91.9 \%$ \\
\hline 117 & $35.8 \%$ & $86.7 \%$ & $35.8 \%$ & $75.1 \%$ & $40.8 \%$ & $50.9 \%$ \\
\hline 69 & $23.9 \%$ & $40.2 \%$ & $23.9 \%$ & $65.2 \%$ & $71.6 \%$ & $48.6 \%$ \\
\hline 67 & $86.1 \%$ & $99.4 \%$ & $86.1 \%$ & $86.3 \%$ & $80.6 \%$ & $91.6 \%$ \\
\hline 12 & $71.5 \%$ & $80.7 \%$ & $71.5 \%$ & $64.6 \%$ & $22.3 \%$ & $85.1 \%$ \\
\hline 16 & $77.5 \%$ & $24.0 \%$ & $77.5 \%$ & $85.7 \%$ & $83.6 \%$ & $70.6 \%$ \\
\hline 71 & $59.6 \%$ & $55.7 \%$ & $59.6 \%$ & $17.8 \%$ & $52.0 \%$ & $45.3 \%$ \\
\hline 18 & $24.5 \%$ & $32.6 \%$ & $24.5 \%$ & $11.8 \%$ & $91.6 \%$ & $80.3 \%$ \\
\hline 75 & $59.8 \%$ & $84.9 \%$ & $59.8 \%$ & $12.0 \%$ & $89.5 \%$ & $93.5 \%$ \\
\hline 96 & $17.9 \%$ & $60.7 \%$ & $17.9 \%$ & $49.8 \%$ & $17.6 \%$ & $91.2 \%$ \\
\hline 94 & $78.7 \%$ & $57.3 \%$ & $78.7 \%$ & $74.5 \%$ & $74.7 \%$ & $11.6 \%$ \\
\hline 92 & $30.7 \%$ & $67.6 \%$ & $30.7 \%$ & $86.7 \%$ & $79.9 \%$ & $37.4 \%$ \\
\hline 98 & $20.1 \%$ & $54.8 \%$ & $20.1 \%$ & $50.9 \%$ & $3.3 \%$ & $84.5 \%$ \\
\hline 99 & $46.7 \%$ & $36.9 \%$ & $46.7 \%$ & $18.7 \%$ & $24.7 \%$ & $11.5 \%$ \\
\hline 101 & $92.5 \%$ & $0.8 \%$ & $92.5 \%$ & $24.7 \%$ & $43.0 \%$ & $22.8 \%$ \\
\hline
\end{tabular}


Table 7. Loading per node for case 2 .

\begin{tabular}{|c|c|c|c|c|c|c|c|}
\hline Node & 0\% DG & $10 \%$ DG & $20-100 \%$ DG & Node & $0 \%$ DG & $10 \%$ DG & $20-100 \%$ DG \\
\hline 1 & $3.0 \%$ & $3.0 \%$ & $15.2 \%$ & 46 & $3.6 \%$ & $3.6 \%$ & $62.0 \%$ \\
\hline 2 & $90.2 \%$ & $90.2 \%$ & $88.5 \%$ & 49 & $16.5 \%$ & $16.5 \%$ & $53.3 \%$ \\
\hline 4 & $91.1 \%$ & $91.1 \%$ & $91.9 \%$ & 53 & $64.4 \%$ & $64.4 \%$ & $1.5 \%$ \\
\hline 6 & $92.5 \%$ & $92.5 \%$ & $99.2 \%$ & 59 & $44.9 \%$ & $44.9 \%$ & $82.2 \%$ \\
\hline 7 & $94.8 \%$ & $94.8 \%$ & $82.6 \%$ & 60 & $30.2 \%$ & $30.2 \%$ & $87.9 \%$ \\
\hline 10 & $97.0 \%$ & $97.0 \%$ & $47.8 \%$ & 61 & $95.7 \%$ & $95.7 \%$ & $97.8 \%$ \\
\hline 14 & $45.2 \%$ & $45.2 \%$ & $98.8 \%$ & 64 & $12.2 \%$ & $12.2 \%$ & $71.5 \%$ \\
\hline 15 & $61.8 \%$ & $61.8 \%$ & $33.0 \%$ & 66 & $62.3 \%$ & $62.3 \%$ & $91.9 \%$ \\
\hline 19 & $84.0 \%$ & $84.0 \%$ & $82.8 \%$ & 117 & $86.7 \%$ & $86.7 \%$ & $50.9 \%$ \\
\hline 20 & $79.1 \%$ & $79.1 \%$ & $84.2 \%$ & 69 & $40.2 \%$ & $40.2 \%$ & $48.6 \%$ \\
\hline 22 & $91.3 \%$ & $91.3 \%$ & $97.1 \%$ & 67 & $99.4 \%$ & $99.4 \%$ & $91.6 \%$ \\
\hline 25 & $91.9 \%$ & $91.9 \%$ & $93.2 \%$ & 12 & $80.7 \%$ & $80.7 \%$ & $85.1 \%$ \\
\hline 26 & $91.7 \%$ & $91.7 \%$ & $94.0 \%$ & 16 & $24.0 \%$ & $24.0 \%$ & $70.6 \%$ \\
\hline 28 & $78.8 \%$ & $78.8 \%$ & $6.7 \%$ & 71 & $55.7 \%$ & $55.7 \%$ & $45.3 \%$ \\
\hline 27 & $54.2 \%$ & $54.2 \%$ & $95.7 \%$ & 18 & $32.6 \%$ & $32.6 \%$ & $80.3 \%$ \\
\hline 29 & $94.7 \%$ & $94.7 \%$ & $64.4 \%$ & 75 & $84.9 \%$ & $84.9 \%$ & $93.5 \%$ \\
\hline 31 & $61.3 \%$ & $61.3 \%$ & $76.7 \%$ & 96 & $60.7 \%$ & $60.7 \%$ & $91.2 \%$ \\
\hline 9 & $39.7 \%$ & $39.7 \%$ & $98.0 \%$ & 94 & $57.3 \%$ & $57.3 \%$ & $11.6 \%$ \\
\hline 34 & $90.4 \%$ & $90.4 \%$ & $71.1 \%$ & 92 & $67.6 \%$ & $67.6 \%$ & $37.4 \%$ \\
\hline 40 & $57.4 \%$ & $57.4 \%$ & $11.2 \%$ & 98 & $54.8 \%$ & $54.8 \%$ & $84.5 \%$ \\
\hline 41 & $88.7 \%$ & $88.7 \%$ & $93.9 \%$ & 99 & $36.9 \%$ & $36.9 \%$ & $11.5 \%$ \\
\hline 45 & $82.5 \%$ & $82.5 \%$ & $6.7 \%$ & 101 & $0.8 \%$ & $0.8 \%$ & $22.8 \%$ \\
\hline 50 & $43.5 \%$ & $43.5 \%$ & $56.9 \%$ & & & & \\
\hline
\end{tabular}

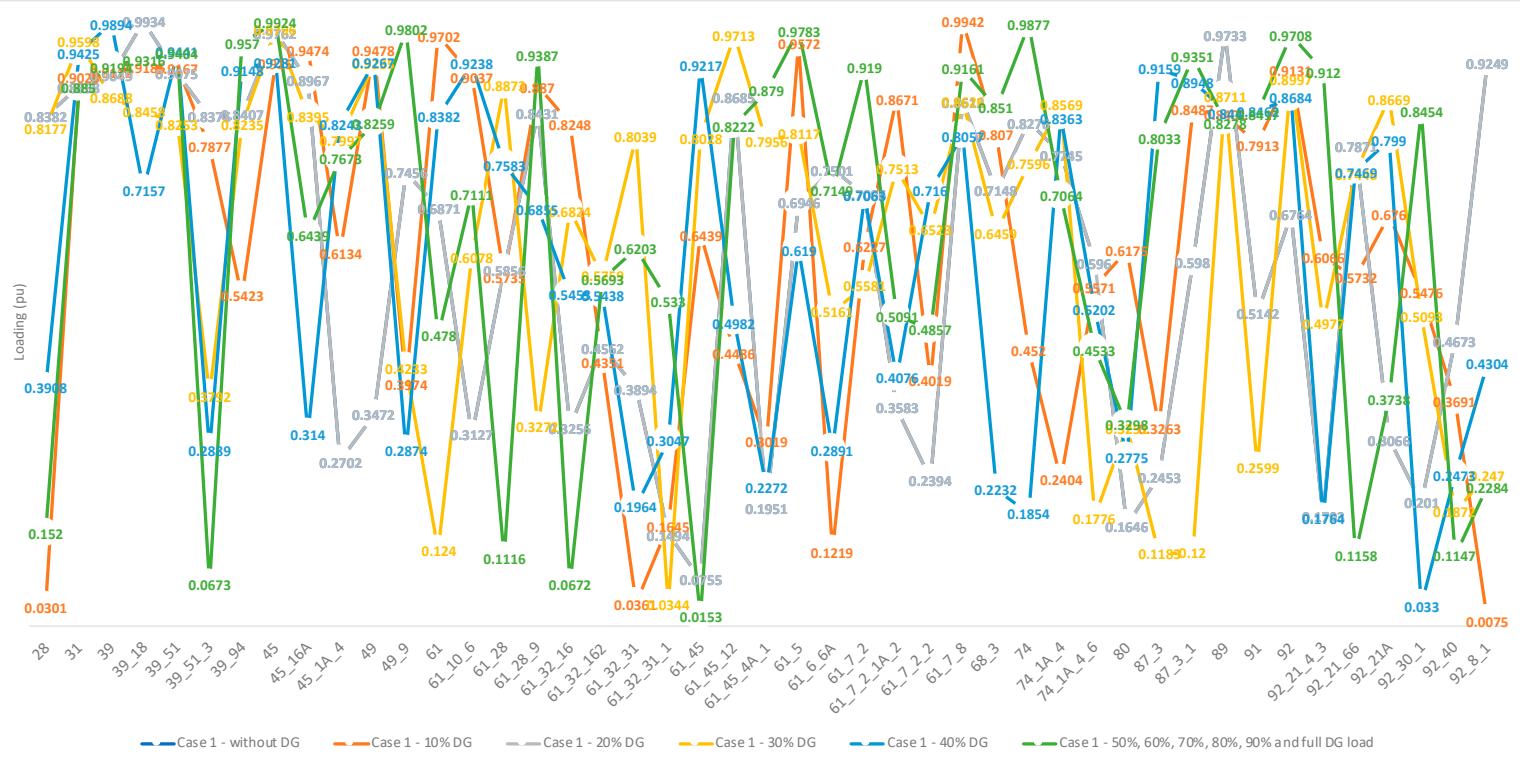

Figure 4. Graphic representation of the loading per node for case 1. 
Table 8. Loading per node for case 3.

\begin{tabular}{|c|c|c|c|c|c|c|}
\hline Node & $0 \%$ DG & $10 \% \mathrm{DG}$ & $20 \%$ DG & $30 \%$ DG & $40 \%$ DG & $50-100 \%$ DG \\
\hline 1 & $3.0 \%$ & $15.2 \%$ & $15.2 \%$ & $15.2 \%$ & $15.2 \%$ & $53.1 \%$ \\
\hline 2 & $90.2 \%$ & $88.5 \%$ & $88.5 \%$ & $88.5 \%$ & $88.5 \%$ & $90.5 \%$ \\
\hline 4 & $91.1 \%$ & $91.9 \%$ & $91.9 \%$ & $91.9 \%$ & $91.9 \%$ & $96.8 \%$ \\
\hline 6 & $92.5 \%$ & $99.2 \%$ & $99.2 \%$ & $99.2 \%$ & $99.2 \%$ & $94.5 \%$ \\
\hline 7 & $94.8 \%$ & $82.6 \%$ & $82.6 \%$ & $82.6 \%$ & $82.6 \%$ & $61.8 \%$ \\
\hline 10 & $97.0 \%$ & $47.8 \%$ & $47.8 \%$ & $47.8 \%$ & $47.8 \%$ & $61.8 \%$ \\
\hline 14 & $45.2 \%$ & $98.8 \%$ & $98.8 \%$ & $98.8 \%$ & $98.8 \%$ & $5.0 \%$ \\
\hline 15 & $61.8 \%$ & $33.0 \%$ & $33.0 \%$ & $33.0 \%$ & $33.0 \%$ & $91.7 \%$ \\
\hline 19 & $84.0 \%$ & $82.8 \%$ & $82.8 \%$ & $82.8 \%$ & $82.8 \%$ & $85.6 \%$ \\
\hline 20 & $79.1 \%$ & $84.2 \%$ & $84.2 \%$ & $84.2 \%$ & $84.2 \%$ & $93.6 \%$ \\
\hline 22 & $91.3 \%$ & $97.1 \%$ & $97.1 \%$ & $97.1 \%$ & $97.1 \%$ & $90.0 \%$ \\
\hline 25 & $91.9 \%$ & $93.2 \%$ & $93.2 \%$ & $93.2 \%$ & $93.2 \%$ & $91.5 \%$ \\
\hline 26 & $91.7 \%$ & $94.0 \%$ & $94.0 \%$ & $94.0 \%$ & $94.0 \%$ & $91.9 \%$ \\
\hline 28 & $78.8 \%$ & $6.7 \%$ & $6.7 \%$ & $6.7 \%$ & $6.7 \%$ & $22.3 \%$ \\
\hline 27 & $54.2 \%$ & $95.7 \%$ & $95.7 \%$ & $95.7 \%$ & $95.7 \%$ & $76.0 \%$ \\
\hline 29 & $94.7 \%$ & $64.4 \%$ & $64.4 \%$ & $64.4 \%$ & $64.4 \%$ & $94.7 \%$ \\
\hline 31 & $61.3 \%$ & $76.7 \%$ & $76.7 \%$ & $76.7 \%$ & $76.7 \%$ & $99.3 \%$ \\
\hline 9 & $39.7 \%$ & $98.0 \%$ & $98.0 \%$ & $98.0 \%$ & $98.0 \%$ & $93.2 \%$ \\
\hline 34 & $90.4 \%$ & $71.1 \%$ & $71.1 \%$ & $71.1 \%$ & $71.1 \%$ & $58.5 \%$ \\
\hline 40 & $57.4 \%$ & $11.2 \%$ & $11.2 \%$ & $11.2 \%$ & $11.2 \%$ & $3.7 \%$ \\
\hline 41 & $88.7 \%$ & $93.9 \%$ & $93.9 \%$ & $93.9 \%$ & $93.9 \%$ & $31.3 \%$ \\
\hline 45 & $82.5 \%$ & $6.7 \%$ & $6.7 \%$ & $6.7 \%$ & $6.7 \%$ & $16.2 \%$ \\
\hline 50 & $43.5 \%$ & $56.9 \%$ & $56.9 \%$ & $56.9 \%$ & $56.9 \%$ & $77.0 \%$ \\
\hline 46 & $3.6 \%$ & $62.0 \%$ & $62.0 \%$ & $62.0 \%$ & $62.0 \%$ & $34.8 \%$ \\
\hline 49 & $16.5 \%$ & $53.3 \%$ & $53.3 \%$ & $53.3 \%$ & $53.3 \%$ & $85.3 \%$ \\
\hline 53 & $64.4 \%$ & $1.5 \%$ & $1.5 \%$ & $1.5 \%$ & $1.5 \%$ & $88.1 \%$ \\
\hline 59 & $44.9 \%$ & $82.2 \%$ & $82.2 \%$ & $82.2 \%$ & $82.2 \%$ & $59.2 \%$ \\
\hline 60 & $30.2 \%$ & $87.9 \%$ & $87.9 \%$ & $87.9 \%$ & $87.9 \%$ & $79.4 \%$ \\
\hline 61 & $95.7 \%$ & $97.8 \%$ & $97.8 \%$ & $97.8 \%$ & $97.8 \%$ & $57.0 \%$ \\
\hline 64 & $12.2 \%$ & $71.5 \%$ & $71.5 \%$ & $71.5 \%$ & $71.5 \%$ & $53.9 \%$ \\
\hline 66 & $62.3 \%$ & $91.9 \%$ & $91.9 \%$ & $91.9 \%$ & $91.9 \%$ & $66.7 \%$ \\
\hline 117 & $86.7 \%$ & $50.9 \%$ & $50.9 \%$ & $50.9 \%$ & $50.9 \%$ & $76.5 \%$ \\
\hline 69 & $40.2 \%$ & $48.6 \%$ & $48.6 \%$ & $48.6 \%$ & $48.6 \%$ & $88.3 \%$ \\
\hline 67 & $99.4 \%$ & $91.6 \%$ & $91.6 \%$ & $91.6 \%$ & $91.6 \%$ & $96.7 \%$ \\
\hline 12 & $80.7 \%$ & $85.1 \%$ & $85.1 \%$ & $85.1 \%$ & $85.1 \%$ & $53.7 \%$ \\
\hline 16 & $24.0 \%$ & $70.6 \%$ & $70.6 \%$ & $70.6 \%$ & $70.6 \%$ & $34.3 \%$ \\
\hline 71 & $55.7 \%$ & $45.3 \%$ & $45.3 \%$ & $45.3 \%$ & $45.3 \%$ & $8.4 \%$ \\
\hline 18 & $32.6 \%$ & $80.3 \%$ & $80.3 \%$ & $80.3 \%$ & $80.3 \%$ & $46.7 \%$ \\
\hline 75 & $84.9 \%$ & $93.5 \%$ & $93.5 \%$ & $93.5 \%$ & $93.5 \%$ & $65.0 \%$ \\
\hline 96 & $60.7 \%$ & $91.2 \%$ & $91.2 \%$ & $91.2 \%$ & $91.2 \%$ & $48.7 \%$ \\
\hline 94 & $57.3 \%$ & $11.6 \%$ & $11.6 \%$ & $11.6 \%$ & $11.6 \%$ & $82.8 \%$ \\
\hline 92 & $67.6 \%$ & $37.4 \%$ & $37.4 \%$ & $37.4 \%$ & $37.4 \%$ & $97.5 \%$ \\
\hline 98 & $54.8 \%$ & $84.5 \%$ & $84.5 \%$ & $84.5 \%$ & $84.5 \%$ & $45.1 \%$ \\
\hline 99 & $36.9 \%$ & $11.5 \%$ & $11.5 \%$ & $11.5 \%$ & $11.5 \%$ & $96.9 \%$ \\
\hline 101 & $0.8 \%$ & $22.8 \%$ & $22.8 \%$ & $22.8 \%$ & $22.8 \%$ & $70.3 \%$ \\
\hline
\end{tabular}


Table 9. Loading per node for case 4 .

\begin{tabular}{cccccccc}
\hline Node & All Cases & Node & All Cases & Node & All Cases & Node & All Cases \\
\hline 1 & $53.1 \%$ & 26 & $91.9 \%$ & 49 & $85.3 \%$ & 71 & $8.4 \%$ \\
2 & $90.5 \%$ & 28 & $22.3 \%$ & 53 & $88.1 \%$ & 18 & $46.7 \%$ \\
4 & $96.8 \%$ & 27 & $76.0 \%$ & 59 & $59.2 \%$ & 75 & $65.0 \%$ \\
6 & $94.5 \%$ & 29 & $94.7 \%$ & 60 & $79.4 \%$ & 96 & $48.7 \%$ \\
7 & $61.8 \%$ & 31 & $99.3 \%$ & 61 & $57.0 \%$ & 94 & $82.8 \%$ \\
10 & $61.8 \%$ & 9 & $93.2 \%$ & 64 & $53.9 \%$ & 92 & $97.5 \%$ \\
14 & $5.0 \%$ & 34 & $58.5 \%$ & 66 & $66.7 \%$ & 98 & $45.1 \%$ \\
15 & $91.7 \%$ & 40 & $3.7 \%$ & 117 & $76.5 \%$ & 99 & $96.9 \%$ \\
19 & $85.6 \%$ & 41 & $31.3 \%$ & 69 & $88.3 \%$ & 101 & $70.3 \%$ \\
20 & $93.6 \%$ & 45 & $16.2 \%$ & 67 & $96.7 \%$ & & \\
22 & $90.0 \%$ & 50 & $77.0 \%$ & 12 & $53.7 \%$ & & \\
25 & $91.5 \%$ & 46 & $34.8 \%$ & 16 & $34.3 \%$ & & \\
\hline
\end{tabular}

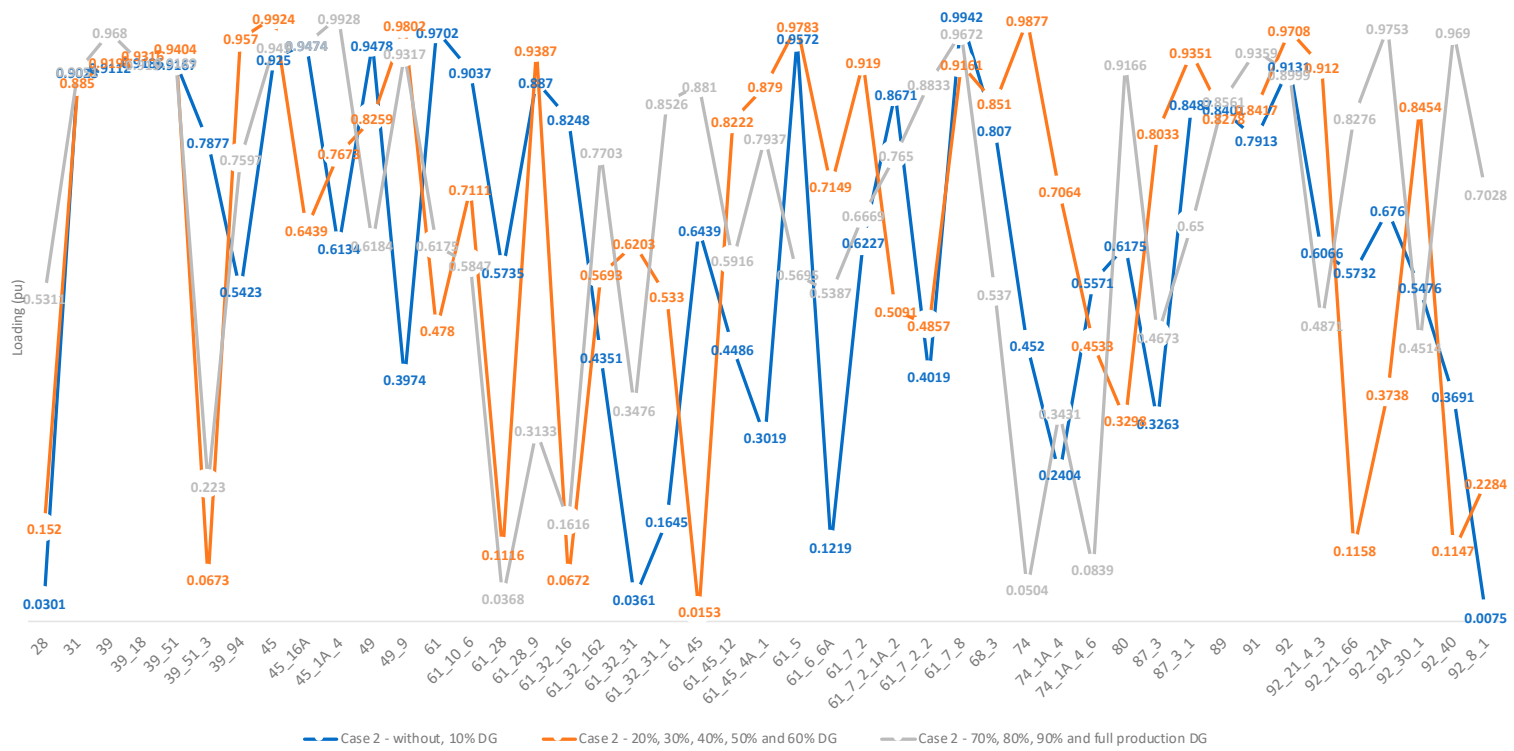

Figure 5. Loading per node according for case 2.

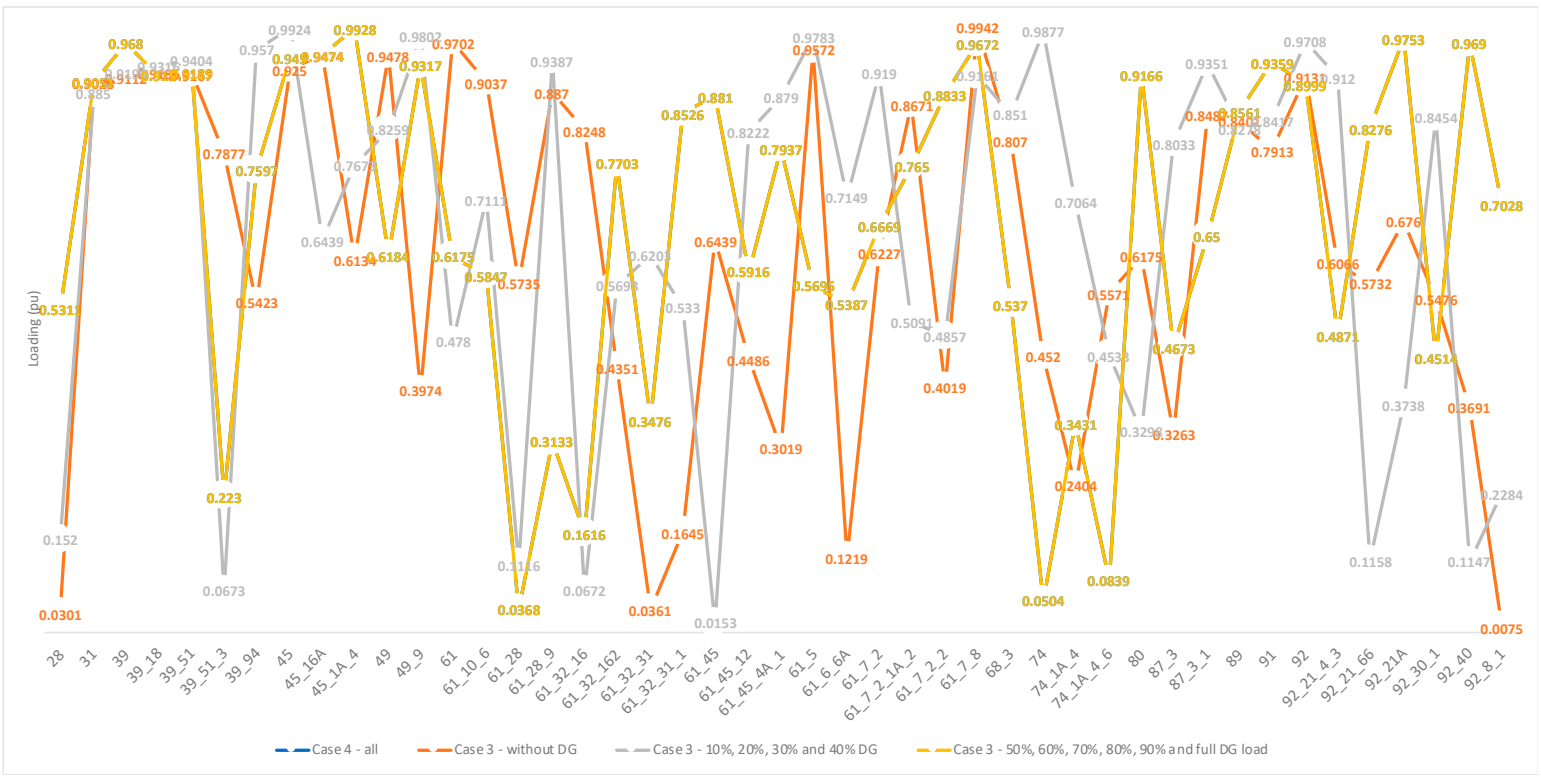

Figure 6. Loading per node for cases 3 and 4. 
Considering case 2 (Table 7 and Figure 5), similar results are being observed. The proposed load for several transformers $(15,40,49,50,69,75,92,94,99,101)$ does not exceed $70 \%$ of their installed power at any production from the renewable energy sources. Similarly, the proposed load of the transformers 1, 40, 46 and 71 for case 3 (Table 8 and Figure 6) and 1, 7, 10, 12, 14, 16, 59, 61, 64, 66 and 98 for case 4 (Table 9 and Figure 4) does not exceed their installed power capacity. Note that these nodes are the points that are expected to receive more attention for downscaling and/or line reinforcing. Added to the above, it is necessary to highlight the impact of the considered distribution generation (DG) on the obtained results. In cases 1 and 3, the loading per node for DG from $50 \%$ to $100 \%$ of the installed capacity does not change. In case 2, DG from $20 \%$ to $100 \%$ provides the same results, since for case 4 , the distribution generation does not affect the proposed loading of each transformer.

The proposed method is able to evaluate the performance of the line after reinforcement, even if this is done using meshed networks configuration. It can be used additionally to any other distribution optimization method. The results are able to propose specific operational points of the line based on the production of the connected distributed generations. In this case, the optimal loading of the line at each of its points is predefined. Given that according to the current operational procedures for the distribution lines it is not possible to control loading with such an accuracy, the applicability of the method is restrained.

The connection of new elements such as electric vehicles and storage, on one hand substantially enhances the capability of demand management and on the other hand there are limitations for lines' expansion. Existing bibliography on the field supports this approach and provides solutions on this direction. Electric vehicles can be aggregated to a virtual power plant, being able to offer ancillary services [40]. This technology can be applied here to always have the operation near the optimal points [41]. However, it has to be mentioned that this procedure would potentially affect customers comfort due to the prioritization of network capacity over user immediate requirements for charging but with substantial benefit to grid flexibility and performance [42].

\section{Conclusions}

This paper proposes an innovative probabilistic method for evaluating the potential performance of meshed and radial distribution lines, improving electric energy distribution grids reinforcement decisions. It is developed as additional to the existing distribution networks optimization methods, offering a tool for performance evaluation. Furthermore, it serves to the creation of operational points to be used for demand management, if electric vehicle charging is completely controlled. This method is a useful tool for network planners, operators and large users, since they are more able to estimate the robustness of a given network after reinforcement and its capability to host electric vehicle loads. The operational points are loading patterns for the transformers of the low voltage distribution network that minimize voltage drop and maximize total loading. They are calculated for increasing production of the connected to the line distributed generators. Other distribution network lines are expected to have similar behavior. They shall present specific operational patterns and their reinforcement could be also evaluated using this method. Future work includes the investigation of the adoption of the appropriate protection schemes that will ensure the reliable and uninterruptable operation of the network.

Author Contributions: Conceptualization, methodology, V.V.; writing-original draft preparation, writing—-review and editing, S.L.; validation, C.A.C.; resources, data curation, G.S.

Funding: The authors acknowledge financial support for the open source publication of this work from the Special Account for Research of ASPETE through the funding program "Strengthening research of ASPETE faculty members" under the project "DECA".

Acknowledgments: The calculation resources for this research were provided by Okeanos high performance cloud computing [33]. This work was also supported by computational time granted from the Greek Research \& Technology Network (GRNET) in the National HPC facility—ARIS—under project ID pa171102 [39]. The authors would like to thank the unknown reviewers, whose comments have improved the quality of this work. 
Conflicts of Interest: The authors declare no conflict of interest.

\section{References}

1. Hadjiionas, S.; Oikonomou, D.; Fotis, G.; Vita, V.; Ekonomou, L.; Pavlatos, C. Green field planning of distribution systems. In Proceedings of the 11th WSEAS International Conference on Automatic Control, Modelling \& Simulation (ACMOS'09), Istanbul, Turkey, 3 May-1 June 2009.

2. Short, T.A. Electric Power Distribution Equipment and Systems; CRC Press, Taylor \& Francis Group: Boca Raton, FL, USA; London, UK; New York, NY, USA, 2014.

3. Alvarez-Herault, M.-C.; N’Doye, N.; Gandioli, C.; Hadjsaid, N.; Tixador, P. Meshed distribution network vs reinforcement to increase the distributed generation connection. Sustain. Energy Grids Netw. 2015, 1, $20-27$. [CrossRef]

4. Novoselnik, B.; Bolfek, M.; Bošković, M.; Baotić, M. Electrical Power Distribution System Reconfiguration: Case Study of a Real-life Grid in Croatia. IFAC-Pap. OnLine 2017, 50, 61-66. [CrossRef]

5. Dumbrava, V.; Miclescu, T.; Lazaroiu, G.C. Power Distribution Networks Planning Optimization in Smart Cities. City Netw. Springer Optim. Appl. 2017, 128, 213-226.

6. Jordehi, A.R. Optimisation of electric distribution systems: A review. Renew. Sustain. Energy Rev. 2015, 51, 1088-1100. [CrossRef]

7. Shuai, W.; Maillé, P.; Pelov, A. Charging Electric Vehicles in the Smart City: A Survey of Economy-Driven Approaches. IEEE Trans. Intell. Transp. Syst. 2016, 17, 2089-2106. [CrossRef]

8. Hu, J.; You, S.; Lind, M.; Østergaard, J. Coordinated Charging of Electric Vehicles for Congestion Prevention in the Distribution Grid. IEEE Trans. Smart Grid 2014, 5, 703-711. [CrossRef]

9. Sundström, O.; Binding, C. Flexible Charging Optimization for Electric Vehicles Considering Distribution Grid Constraints. IEEE Trans. Smart Grid 2012, 3, 26-37. [CrossRef]

10. Mojdehi, M.N.; Ghosh, P. An On-Demand Compensation Function for an EV as a Reactive Power Service Provider. IEEE Trans. Veh. Technol. 2016, 65, 4572-4583. [CrossRef]

11. Beaude, O.; Lasaulce, S.; Hennebel, M.; Mohand-Kaci, I. Reducing the Impact of EV Charging Operations on the Distribution Network. IEEE Trans. Smart Grid 2016, 7, 2666-2679. [CrossRef]

12. Sato, T.; Kammen, D.M.; Duan, B.; Macuha, M.; Zhou, Z.; Wu, J.; Tariq, M.; Asfaw, S.A. Smart Grid Standards Specifications, Requirements and Technologies; John Wiley \& Sons Singapore Pte. Ltd.: Singapore, 2015.

13. Daina, N.; Sivakumar, A.; Polak, J.W. Electric vehicle charging choices: Modelling and implications for smart charging services. Transp. Res. Part C 2017, 81, 36-56. [CrossRef]

14. Weiller, C.; Neely, A. Using electric vehicles for energy services: Industry perspectives. Energy 2014, 77, 194-200. [CrossRef]

15. Schmidt, J.; Lauven, L.-P.; Ihle, N.; Kolbe, L.M. Demand side integration for electric transport vehicles. Int. J. Energy Sector Manag. 2015, 9, 471-495. [CrossRef]

16. Peng, C.; Zou, J.; Lian, L. Dispatching strategies of electric vehicles participating in frequency regulation on power grid: A review. Renew. Sustain. Energy Rev. 2017, 68, 147-152. [CrossRef]

17. Pavic, I.; Capuder, T.; Kuzle, I. A Comprehensive Approach for Maximizing Flexibility Benefits of Electric Vehicles. IEEE Syst. J. 2017, 12, 2882-2893. [CrossRef]

18. Vita, V. Development of a decision-making algorithm for the optimum size and placement of distributed generation units in distribution networks. Energies 2017, 10, 1433. [CrossRef]

19. Zubo, R.; Mokryani, G.; Rajamani, H.-S.; Aghaei, J.; Niknam, T.; Pillai, P. Operation and planning of distribution networks with integration of renewable distributed generators considering uncertainties: A review. Renew. Sustain. Energy Rev. 2017, 72, 1177-1198. [CrossRef]

20. Singh, B.; Sharma, J. A review on distributed generation planning. Renew. Sustain. Energy Rev. 2017, 76, 529-544. [CrossRef]

21. Ehsan, A.; Yang, Q. Optimal integration and planning of renewable distributed generation in the power distribution networks: A review of analytical techniques. Appl. Energy 2018, 210, 44-59. [CrossRef]

22. Singh, B.; Mishra, D.K. A survey on enhancement of power system performances by optimally placed DG in distribution networks. Energy Rep. 2018, 4, 129-158. [CrossRef]

23. Shang, Y. Resilient Multiscale Coordination Control against Adversarial Nodes. Energies 2018, 11, 1844. [CrossRef] 
24. Sedghi, M.; Ahmadian, A.; Aliakbar-Golkar, M. Assessment of optimization algorithms capability in distribution network planning: Review, comparison and modification techniques. Renew. Sustain. Energy Rev. 2016, 66, 415-434. [CrossRef]

25. Kalos, M.H.; Whitlock, P.A. Monte Carlo Methods; WILEY-VCH Verlag GmbH \& Co. KGaA: Weinheim, Germany, 2004.

26. Marmidis, G.; Lazarou, S.; Pyrgioti, E. Optimal placement of wind turbines in a wind park using Monte Carlo simulation. Renew. Energy 2008, 33, 1455-1460. [CrossRef]

27. Banerjee, B.; Jayaweera, D.; Islam, S. Modelling and Simulation of Power Systems. In Smart Power Systems and Renewable Energy System Integration; Springer: Cham, Switzerland, 2016; pp. 15-28.

28. Prusty, B.R.; Jena, D. A critical review on probabilistic load flow studies in uncertainty constrained power systems with photovoltaic generation and a new approach. Renew. Sustain. Energy Rev. 2017, 69, 1286-1302. [CrossRef]

29. Mokryani, G. Active distribution networks planning with integration of demand response. Sol. Energy 2015, 122, 1362-1370. [CrossRef]

30. Hemmati, R.; Hooshmand, R.-A.; Taheri, N. Distribution network expansion planning and DG placement in the presence of uncertainties. Electr. Power Energy Syst. 2015, 73, 665-673. [CrossRef]

31. Shang, Y. Optimal Control Strategies for Virus Spreading in Inhomogeneous Epidemic Dynamics. Can. Math. Bull. 2013, 56, 621-629. [CrossRef]

32. Piao, M.; Li, Y.; Huang, G. Development of a stochastic simulation-optimization model for planning electric power systems-A case study of Shanghai, China. Energy Convers. Manag. 2014, 86, 111-124. [CrossRef]

33. Okeanos High Performance Cloud Computing, Greek Research and Technology Network (GRNET). 2017. Available online: https://okeanos-global.grnet.gr/ (accessed on 2 December 2018).

34. Lazarou, S.; Vita, V.; Ekonomou, L. An open data repository for steady state analysis of a 100-node electricity distribution network with moderate connection of renewable energy sources. Data Brief 2018, 16, 1095-1101. [CrossRef]

35. Zimmerman, R.D.; Murillo-Sanchez, C.E. Matpower 6.0 User's Manual. 2016. Available online: http: //www.pserc.cornell.edu/matpower/manual.pdf (accessed on 9 November 2018).

36. Zimmerman, R.D.; Murillo-Sánchez, C.E.; Thomas, R.J. MATPOWER: Steady-State Operations, Planning and Analysis Tools for Power Systems Research and Education. IEEE Trans. Power Syst. 2011, 26, 12-19. [CrossRef]

37. Murillo-Sánchez, C.E.; Zimmerman, R.D.; Anderson, C.L.; Thomas, R.J. Secure Planning and Operations of Systems with Stochastic Sources, Energy Storage and Active Demand. IEEE Trans. Smart Grid 2013, 4, 2220-2229. [CrossRef]

38. Matlab Runtime. 2017. Available online: https://www.mathworks.com/products/compiler/matlab-runtime. html (accessed on 9 March 2019).

39. Aris High Performance Computing, Greek Research and Technology Network (GRNET). 2017. Available online: https://hpc.grnet.gr/ (accessed on 17 March 2019).

40. Zakariazadeh, A.; Jadid, S.; Siano, P. Integrated operation of electric vehicles and renewable generation in a smart distribution system. Energy Convers. Manag. 2015, 89, 99-110. [CrossRef]

41. Bhattarai, B.P.; Myers, K.S.; Bak-Jensen, B.; Mendaza, I.D.; Turk, R.J.; Gentle, J.P. Optimum aggregation of geographically distributed flexible resources in strategic smart-grid/microgrid locations. Electr. Power Energy Syst. 2017, 92, 193-201. [CrossRef]

42. Knezović, K.; Marinelli, M.; Zecchino, A.; Andersen, P.B.; Traeholt, C. Supporting involvement of electric vehicles in distribution grids: Lowering the barriers for a proactive integration. Energy 2017, 134, 458-468. [CrossRef]

(C) 2019 by the authors. Licensee MDPI, Basel, Switzerland. This article is an open access article distributed under the terms and conditions of the Creative Commons Attribution (CC BY) license (http://creativecommons.org/licenses/by/4.0/). 